\title{
Estimating the Reliability Function for a Family of Exponentiated Distributions
}

\author{
Ajit Chaturvedi and Anupam Pathak \\ Department of Statistics, University of Delhi, Delhi 110007, India \\ Correspondence should be addressed to Anupam Pathak; pathakanupam24@gmail.com
}

Received 4 June 2013; Revised 17 December 2013; Accepted 22 March 2014; Published 29 April 2014

Academic Editor: Dejian Lai

Copyright (c) 2014 A. Chaturvedi and A. Pathak. This is an open access article distributed under the Creative Commons Attribution License, which permits unrestricted use, distribution, and reproduction in any medium, provided the original work is properly cited.

\begin{abstract}
A family of exponentiated distributions is proposed. The problems of estimating the reliability function are considered. Uniformly minimum variance unbiased estimators and maximum likelihood estimators are derived. A comparative study of the two methods of estimation is done. Simulation study is preformed.
\end{abstract}

\section{Introduction}

The reliability function $R(t)$ is defined as the probability of failure-free operation until time $t$. Thus, if the random variable (rv) $X$ denotes the lifetime of an item, then $R(t)=$ $P(X>t)$. Another measure of reliability under stressstrength setup is the probability $P=P(X>Y)$, which represents the reliability of an item of random strength $X$ subject to random stress $Y$.

A lot of work has been done in the literature to deal with inferential problems related to various exponentiated distributions. In particular, Mudholkar and Srivastava [1] considered exponentiated Weibull family for analyzing bathtub failure-real data. The exponentiated Weibull family was also applied to the bus-motor-failure data in Davis [2], to head-and-neck cancer clinical trial data in Efron [3] [see [4]], and in analyzing flood data [see [5]]. Jiang and Murthy [6] considered exponentiated Weibull family and illustrated some of its properties by using graphical approach. Nassar and Eissa [7, 8] studied a two-parameter exponentiated Weibull distribution and they gave some of its properties and estimated the parameters by using the maximum likelihood and Bayes methods based on type II censored data. They used the squared-error and linear in exponential (LINEX) loss functions and an informative prior to obtain the Bayes estimates. Pal et al. [9] showed that the failure rate of exponentiated Weibull behaves more like the failure rate of the Weibull distribution than that of the
Gamma distribution. They also obtained maximum likelihood estimators, Fisher's information matrix, and confidence intervals for related parameters. Gupta et al. [10] introduced exponentiated exponential distribution in a series of papers. Gupta and Kundu [11-17], Gupta et al. [18], and Kundu et al. [19] concentrated on the study of the exponentiated exponential distribution. Kundu and Gupta [20] stated that exponentiated exponential distribution is an alternative to the well-known two-parameter Gamma, two-parameter Weibull or two-parameter lognormal distributions. Kundu and Gupta [20] estimated the reliability of the stress-strength model $P(X<Y)$, when $X$ and $Y$ are independent exponentiated exponential random variables. Raqab and Ahsanullah [21] estimated the parameters of the exponentiated exponential based on ordered statistics. Inference for exponentiated Weibull distribution based on record values was made by Raqab [22]. Abdel-Hamid and AL-Hussaini [23] obtained the maximum likelihood estimates of the parameters when step-stress accelerated life testing is applied for exponentiated exponential distribution. Al-Hussaini and Hussein [24, 25] fitted the exponentiated Burr model to the data of breaking strength of single carbon fibers of particular length (data in [26]) in the complete sample case and when type II censoring is imposed on data. SELF and LINEX loss functions are used in the Bayes estimation. Gupta et al. [10] introduced a new distribution, called the exponentiated Pareto distribution. Shawky and Abu-Zinadah [27] obtained maximum likelihood estimators of the different parameters of the 
exponentiated Pareto distribution. They also considered five other estimation procedures and compared their performances through numerical simulations.

The purpose of the present paper is manyfold. We propose a family of exponentiated distributions, which covers as many as ten exponentiated distributions as specific cases. We consider the problems of estimating $R(t)$ and " $P$." Uniformly minimum variance unbiased estimators (UMVUES) and maximum likelihood estimators (MLES) are derived. In order to obtain these estimators, the major role is played by the estimator of the probability density function (pdf) at a specified point, and the functional forms of the parametric functions to be estimated are not needed. It is worth mentioning here that, in order to estimate " $P$," in the literature, the authors have considered the case when $X$ and $Y$ follow the same distributions, may be with different parameters. We have generalized the result to the case when $X$ and $Y$ may follow any distribution from the proposed exponentiated family of distributions. Simulation study is carried out to investigate the performance of the estimators.

In Section 2, we introduce the family of exponentiated distributions. In Section 3, we derive the UMVUES of $R(t)$ and " $P$." In Section 4, we obtain the MLES of $R(t)$ and " $P$." In Section 5 , analysis of a simulated data has been presented for illustrative purposes. Finally, in Section 6, conclusions have been provided.

\section{The Family of Exponentiated Distributions}

Let the $\mathrm{rv} X$ follow the distribution having the pdf

$$
\begin{array}{r}
f(x ; a, \alpha, \lambda, \underline{\theta})=\alpha \lambda g^{\prime}(x ; a, \underline{\theta}) e^{-\lambda g(x ; a, \underline{\theta})}\left(1-e^{-\lambda g(x ; a, \underline{\theta})}\right)^{\alpha-1} ; \\
x>a \geq 0, \alpha, \lambda>0 .
\end{array}
$$

Here, $g(x ; a, \underline{\theta})$ is a function of $X$ and may also depend on (may be vector-valued) parameter $\underline{\theta}$. Moreover, $g(x ; a, \underline{\theta})$ is a real-valued, monotonically increasing function of $X$ with $g(a ; a, \underline{\theta})=0, g(\infty ; a, \underline{\theta})=\infty$, and $g^{\prime}(x ; a, \underline{\theta})$ denotes the derivative of $g(x ; a, \bar{\theta})$ with respect to $x$. Our case is a special model of the beta extended Weibull family proposed by Cordeiro et al. [28] and can be obtained from equation (7) when $b=0$. We note that (1) represents a family of exponentiated distributions, as it covers the following exponentiated distributions as special cases.

(i) For $g(x ; a, \underline{\theta})=x$ and $a=0$, we get the exponentiated exponential distribution [see [10]].

(ii) For $g(x ; a, \underline{\theta})=x^{2}$ and $a=0$, it gives exponentiated Rayleigh distribution [see [29]].

(iii) For $g(x ; a, \underline{\theta})=x^{\delta}, \delta>0$ and $a=0$, we get the exponentiated Weibull distribution [see [1]].

(iv) For $g(x ; a, \underline{\theta})=\log \left(1+x^{\delta}\right), \delta>0$ and $a=0$, it leads us to pdf of exponentiated Burr distribution [see [24]].

(v) For $g(x ; a, \underline{\theta})=\log (x / a)$, it turns out to be exponentiated Pareto distribution [see [10]]. (vi) For $g(x ; a, \underline{\theta})=(1+(x / \xi)), \xi>0$ and $a=0$, it gives us exponentiated Lomax distribution [see [30]].

(vii) For $g(x ; a, \underline{\theta})=\left(1+\left(x^{\xi} / \nu\right)\right), \xi>0, v>0$ and $a=0$, it turns out to be exponentiated Burr distribution with scale parameter $\nu$ [see [31]].

(viii) For $g(x ; a, \underline{\theta})=x^{\gamma} \exp (\nu x), \gamma>0, v>0$ and $a=0$, it gives the exponentiated form of modified Weibull distribution of Lai et al. [32].

(ix) For $g(x ; a, \underline{\theta})=\gamma \exp \left[(x / \gamma)^{\nu}-1\right], \gamma>0, \nu>0$ and $a=$ 0 , we get the exponentiated form of a modification of Weibull distribution [see [33]].

(x) For $g(x ; a, \underline{\theta})=(x-a)+(\nu / \lambda) \log ((x+\nu) /(a+\nu))$, $\nu>0$, it gives exponentiated form of the generalized Pareto distribution [see [34]].

\section{UMVUES of $R(t)$ and " $P$ "}

Throughout this section, we assume that $\alpha$ is unknown, but " $a$ ", $\lambda$, and $\underline{\theta}$ are known. Let $X_{1}, X_{2}, \ldots, X_{n}$ be a random sample of size $n$ from (1).

Lemma 1. Let $T=\sum_{i=1}^{n} \log \left(1-e^{-\lambda g\left(X_{i}, a, \underline{\theta}\right)}\right)$. Then, $T$ is complete and sufficient for the family of distributions given in (1). Moreover, the pdf of $T$ is

$$
g(t ; \alpha, \lambda, \underline{\theta})=\frac{\alpha^{n}}{\Gamma(n)}(-t)^{n-1} e^{\alpha t} ; \quad-\infty<t<0 .
$$

Proof. Denoting by $h\left(x_{1}, x_{2}, \ldots, x_{2} ; a, \alpha, \lambda, \underline{\theta}\right)$ the joint pdf of $X_{1}, X_{2}, \ldots, X_{n}$, we have

$$
\begin{aligned}
& h\left(x_{1}, x_{2}, \ldots, x_{2} ; a, \alpha, \lambda, \underline{\theta}\right) \\
& =(\alpha \lambda)^{n} \prod_{i=1}^{n} g^{\prime}\left(x_{i} ; a, \theta\right) e^{-\lambda \sum_{i=1}^{n} g\left(x_{i} ; a, \theta\right)} \\
& \quad \times e^{(\alpha-1) \sum_{i=1}^{n} \log \left(1-e^{-\lambda g\left(x_{i} ; a, \theta\right)}\right)} .
\end{aligned}
$$

It follows from (3) and Fishers-Neyman factorization theorem [see [35, p. 341]] that $T$ is sufficient for the family of distributions $f(x ; a, \alpha, \lambda, \underline{\theta})$. It is easy to see that the rv $V=-2 \alpha \log \left(1-e^{-\bar{\lambda} g(x ; a, \underline{\theta})}\right)$ follows $\chi_{(2)}^{2}$ distribution. Thus, from the well-known additive property of Chi-square distribution [see Johnson and Kotz, [36, p. 170]], $-2 \alpha T=-2 \alpha \sum_{i=1}^{n} \log \left(1-e^{-\lambda g\left(x_{i} ; a, \underline{\theta}\right)}\right)$ is an $\chi_{(2 n)}^{2}$ rv and the result follows. Since the distribution of $T$ belongs to exponential family, it is also complete [see [35, p. 341]].

The following lemma provides the UMVUES of the powers of $\alpha$.

Lemma 2. For $q \in(-\infty, \infty)$, the UMVUE of $\alpha^{q}$ is

$$
\widehat{\alpha}^{q}= \begin{cases}\frac{\Gamma(n)}{\Gamma(n-q)}(-T)^{-q} ; & q<n, \\ 0 ; & \text { otherwise. }\end{cases}
$$


Proof. The result follows from Lemma 1, Lehmann-Scheffé theorem [see [35, p. 357]], and the fact that

$$
E(-2 \alpha T)^{-q}=\frac{\Gamma(n-q)}{2^{q} \Gamma(n)} ; \quad q<n .
$$

In the following lemma, we provide the UMVUE of the sampled pdf (1) at a specified point " $x$."

Lemma 3. The UMVUE of $f(x ; a, \alpha, \lambda, \underline{\theta})$ at a specified point " $x$ " is

$\widehat{f}(x ; a, \alpha, \lambda, \underline{\theta})$

$=\left\{\begin{array}{rlr}- & (n-1) \lambda T^{-1}\left(1-e^{-\lambda g(x ; a, \underline{\theta})}\right)^{-1} & \\ & \times g^{\prime}(x ; a, \underline{\theta}) e^{-\lambda g(x ; a, \underline{\theta})} & \\ \quad \times\left[1-T^{-1} \log \left(1-e^{-\lambda g(x ; a, \underline{\theta})}\right)\right]^{n-2} ; & & T<\log \left\{1-e^{-\lambda g(x ; a, \underline{\theta})}\right\}, \\ 0 ; & & \text { otherwise. }\end{array}\right.$

Proof. Since $T$ is complete and sufficient for the family of distributions $f(x ; a, \alpha, \lambda, \underline{\theta})$, any function $H(T)$ of $T$ satisfying $E[H(T)]=f(x ; a, \alpha, \lambda, \underline{\theta})$ will be the UMVUE of $f(x ; a, \alpha, \lambda, \underline{\theta})$. To this end, from (1) and Lemma 1 ,

$$
\begin{aligned}
& \int_{-\infty}^{0} H(t) \frac{\alpha^{n}}{\Gamma(n)}(-t)^{n-1} e^{\alpha t} d t \\
& =\alpha \lambda g^{\prime}(x ; a, \theta) e^{-\lambda g(x ; a, \theta)}\left(1-e^{-\lambda g(x ; a, \theta)}\right)^{\alpha-1},
\end{aligned}
$$

or

$$
\begin{gathered}
-\frac{\alpha^{n-1}}{\Gamma(n)} \int_{\log \left(1-e^{-\lambda g(x ; a, \theta)}\right)}^{\infty} H\left[-u+\log \left\{1-e^{-\lambda g(x ; a, \theta)}\right\}\right] \\
\times\left[-u+\log \left(1-e^{-\lambda g(x ; a, \theta)}\right)\right]^{n-1} e^{-\alpha u} d u \\
=\lambda\left(1-e^{-\lambda g(x ; a, \theta)}\right)^{-1} g^{\prime}(x ; a, \theta) e^{-\lambda g(x ; a, \theta)} .
\end{gathered}
$$

Equation (8) is satisfied if we choose

$$
\begin{aligned}
& H\left[-u+\log \left(1-e^{-\lambda g(x ; a, \underline{\theta})}\right)\right]
\end{aligned}
$$

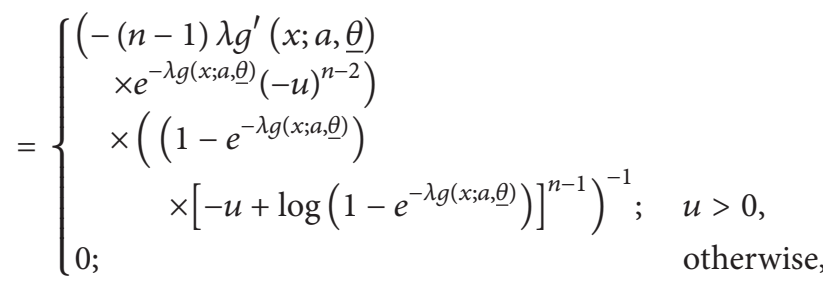

and the lemma holds.

Remark 4. We can write (1) as

$$
\begin{aligned}
f(x ; a, \alpha, \lambda, \underline{\theta})= & \lambda\left(1-e^{-\lambda g(x ; a, \underline{\theta})}\right)^{-1} \\
& \times g^{\prime}(x ; a, \underline{\theta}) e^{-\lambda g(x ; a, \underline{\theta})} \alpha \\
& \times \sum_{i=0}^{\infty} \frac{\left[\log \left(1-e^{-\lambda g(x ; a, \underline{\theta})}\right)\right]^{i} \alpha^{i}}{i !} .
\end{aligned}
$$

Using Lemma 1 of Chaturvedi and Tomer [37] and Lemma 2, the UMVUE of $f(x ; a, \alpha, \lambda, \underline{\theta})$ at a specified point " $x$ " is

$$
\begin{aligned}
\widehat{f}(x ; a, \alpha, \lambda, \underline{\theta})= & \lambda\left(1-e^{-\lambda g(x ; a, \underline{\theta})}\right)^{-1} \\
& \times g^{\prime}(x ; a, \underline{\theta}) e^{-\lambda g(x ; a, \underline{\theta})} \\
& \times \sum_{i=0}^{\infty} \frac{\left[\log \left(1-e^{-\lambda g(x ; a, \underline{\theta})}\right)\right]^{i}}{i !} \widehat{\alpha}^{i+1} \\
= & \lambda\left(1-e^{-\lambda g(x ; a, \underline{\theta})}\right)^{-1} g^{\prime}(x ; a, \underline{\theta}) e^{-\lambda g(x ; a, \underline{\theta})} \\
& \times(-T)^{-1} \sum_{i=0}^{n-2} \frac{\Gamma(n-1)}{\Gamma(n-i-1)}(-T)^{i} \\
& \times \frac{\left[\log \left(1-e^{-\lambda g(x ; a, \underline{\theta})}\right)\right]^{i}}{i !}
\end{aligned}
$$

or

$\widehat{f}(x ; a, \alpha, \lambda, \underline{\theta})$

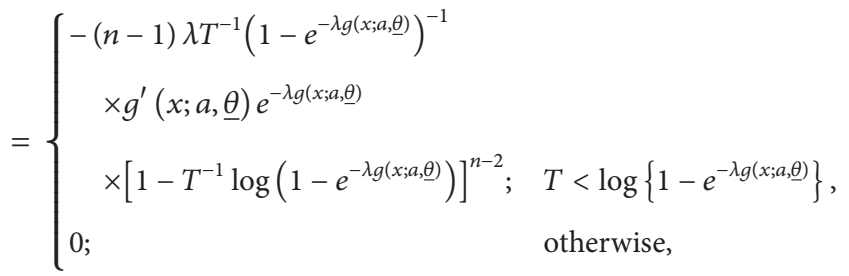

which coincides with Lemma 3. Thus, the UMVUE of the power of $\alpha$ can be used to derive the UMVUE of $\widehat{f}(x ; a, \alpha, \lambda, \underline{\theta})$ at a specified point.

In the following theorem, we obtain the UMVUE of $R(t)$.

Theorem 5. The UMVUE of $R(t)$ is given by

$$
\widehat{R}(t)=\left\{\begin{array}{l}
1-\left[1-T^{-1} \log \left\{1-e^{-\lambda g(t ; a, \underline{\theta})}\right\}\right]^{n-1} ; \\
T<\log \left(1-e^{-\lambda g(t ; a, \underline{\theta})}\right), \\
0 ; \quad \text { otherwise. }
\end{array}\right.
$$

Proof. Let us consider the expected value of the integral $\int_{t}^{\infty} \widehat{f}(x ; a, \alpha, \lambda, \underline{\theta}) d x$ with respect to $T$; that is,

$$
\begin{aligned}
\int_{0}^{\infty} & \left\{\int_{t}^{\infty} \widehat{f}(x ; a, \alpha, \lambda, \underline{\theta}) d x\right\} h(t ; \alpha, \lambda, \underline{\theta}) d t \\
= & \int_{t}^{\infty}\left[E_{T}\{\widehat{f}(x ; a, \alpha, \lambda, \underline{\theta})\}\right] d x \\
= & \int_{t}^{\infty}\{f(x ; a, \alpha, \lambda, \underline{\theta})\} d x=R(t) .
\end{aligned}
$$


We conclude from (14) that the UMVUE of $R(t)$ can be obtained simply integrating $\widehat{f}(x ; a, \alpha, \lambda, \underline{\theta})$ from $t$ to $\infty$. Thus, denoting by $g^{-1}(\cdot)$, the inverse function of $g(x ; a, \underline{\theta})$, from Lemma 3, we obtain

$$
\begin{gathered}
\widehat{R}(t)=-(n-1) T^{-1} \int_{t}^{g^{-1}\left\{-\lambda^{-1} \log \left(1-e^{T}\right)\right\}} \lambda\left(1-e^{-\lambda g(x ; a, \underline{\theta})}\right)^{-1} g^{\prime}(x ; a, \underline{\theta}) e^{-\lambda g(x ; a, \underline{\theta})} \\
\times\left[1-T^{-1} \log \left(1-e^{-\lambda g(x ; a, \underline{\theta})}\right)\right]^{n-2} d x ; \\
T<\log \left\{1-e^{-\lambda g(x ; a, \underline{\theta})}\right\}=-(n-1) \int_{T^{-1} \log \left(1-e^{-\lambda g(t ; a, \theta)}\right)}^{1}(1-y)^{n-2} d y,
\end{gathered}
$$

and the theorem follows.

Let $X$ and $Y$ be two independent rvs following the families of distributions $f_{1}\left(x ; a_{1}, \alpha_{1}, \lambda_{1}, \underline{\theta}_{1}\right)$ and $f_{2}\left(y ; a_{2}, \alpha_{2}, \lambda_{2}, \underline{\theta}_{2}\right)$, respectively. We assume that $\alpha_{1}$ and $\alpha_{2}$ are unknown but $a_{1}, a_{2}, \lambda_{1}, \lambda_{2}, \underline{\theta}_{1}$, and $\underline{\theta}_{2}$ are known. Let $X_{1}, X_{2}, \ldots, X_{n}$ be a random sample of size $n$ from $f_{1}\left(x ; a_{1}, \alpha_{1}, \lambda_{1}, \underline{\theta}_{1}\right)$ and let $Y_{1}, Y_{2}, \ldots, Y_{m}$ be a random sample of size $m$ from $f_{2}\left(y ; a_{2}, \alpha_{2}, \lambda_{2}, \underline{\theta}_{2}\right)$. Let us denote by $T=$ $\sum_{i=1}^{n} \log \left(1-e^{-\lambda_{1} g\left(x_{i} ; a_{1}, \underline{\theta}_{1}\right)}\right)$ and $S=\sum_{j=1}^{m} \log \left(1-e^{-\lambda_{2} h\left(y_{j} ; a_{2}, \theta_{2}\right)}\right)$. In what follows, we obtain the UMVUE of " $P$ ".

Theorem 6. The UMVUE of " $P$ " is given by

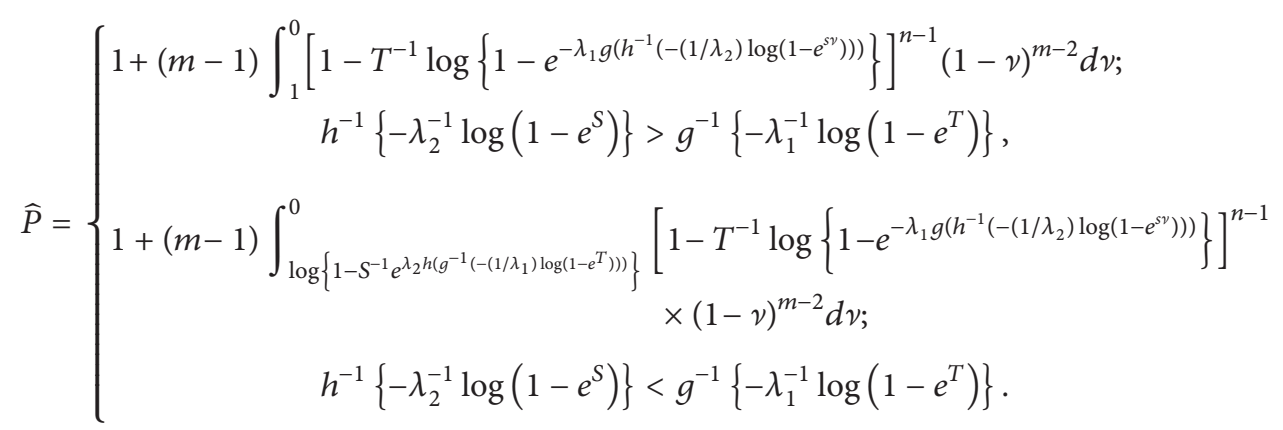

Proof. From the arguments similar to those adopted in proving Theorem 5, it can be shown that the UMVUE of " $P$ " is given by

$$
\widehat{P}=\int_{y=a_{2}}^{\infty} \int_{x=y}^{\infty} \widehat{f}_{1}\left(x ; a_{1}, \alpha_{1}, \lambda_{1}, \underline{\theta}_{1}\right) \hat{f}_{2}\left(y ; a_{2}, \alpha_{2}, \lambda_{2}, \underline{\theta}_{2}\right) d x d y .
$$

Thus, using Lemma 3 ,

$$
\begin{aligned}
\widehat{P}=-\int_{y=a_{2}}^{\infty}\left[\int_{x=y}^{\infty}(n-1) \lambda_{1}\left(1-e^{-\lambda_{1} g\left(x ; a_{1}, \underline{\theta}_{1}\right)}\right)^{-1} g^{\prime}\left(x ; a_{1}, \underline{\theta}_{1}\right) e^{-\lambda_{1} g\left(x ; a_{1}, \underline{\theta}_{1}\right)}\right. \\
\left.\cdot\left(1-T^{-1} \log \left(1-e^{-\lambda_{1} g\left(x ; a_{1}, \underline{\theta}_{1}\right)}\right)\right)^{n-2} d x\right] \cdot \widehat{f}_{2}\left(y ; a_{2}, \alpha_{2}, \lambda_{2}, \underline{\theta}_{2}\right) d y \\
=1+(m-1) \lambda_{2} S^{-1} \int_{y=\max \left[h^{-1}\left\{-\lambda_{2}^{-1} \log \left(1-e^{S}\right)\right\}, g^{-1}\left\{-\lambda_{1}^{-1} \log \left(1-e^{T}\right)\right\}\right]}^{\infty}\left\{1-T^{-1} \log \left(1-e^{-\lambda_{1} g\left(y ; a_{1}, \underline{\theta}_{1}\right)}\right)\right\}^{n-1} \\
\cdot\left(1-e^{-\lambda_{2} h\left(y ; a_{2}, \underline{\theta}_{2}\right)}\right)^{-1} h^{\prime}\left(y ; a_{2}, \underline{\theta}_{2}\right) e^{-\lambda_{2} h\left(y ; a_{2}, \underline{\theta}_{2}\right)} \\
\cdot\left[1-S^{-1} \log \left(1-e^{-\lambda_{2} h\left(y ; a_{2}, \underline{\theta}_{2}\right)}\right)\right]^{m-2} d y .
\end{aligned}
$$

Let us first consider the case when $h^{-1}\left\{-\lambda_{2}^{-1} \log \left(1-e^{S}\right)\right\}>$ $g^{-1}\left\{-\lambda_{1}^{-1} \log \left(1-e^{T}\right)\right\}$. In this case, from (18),

$$
\widehat{P}=1+(m-1) \int_{1}^{0}\left[1-T^{-1} \log \left\{1-e^{-\lambda_{1} g\left(h^{-1}\left(-\left(1 / \lambda_{2}\right) \log \left(1-e^{s \nu}\right)\right)\right)}\right\}\right]^{n-1}(1-\nu)^{m-2} d \nu .
$$


Now we consider the case when $h^{-1}\left\{-\lambda_{2}^{-1} \log \left(1-e^{S}\right)\right\}<$ $g^{-1}\left\{-\lambda_{1}^{-1} \log \left(1-e^{T}\right)\right\}$. In this case, from (18),

$$
\widehat{P}=1+(m-1) \int_{\log \left\{1-S^{-1} e^{\lambda_{2} h\left(g^{-1}\left(-\left(1 / \lambda_{1}\right) \log \left(1-e^{T}\right)\right)\right)}\right\}}^{0}\left[1-T^{-1} \log \left\{1-e^{-\lambda_{1} g\left(h^{-1}\left(-\left(1 / \lambda_{2}\right) \log \left(1-e^{s v}\right)\right)\right)}\right\}\right]^{n-1}(1-\nu)^{m-2} d \nu .
$$

The theorem now follows on combining (19) and (20).

Corollary 7. For the case when $a_{1}=a_{2}=a$, say, $\lambda_{1}=\lambda_{2}=\lambda$, say, $\underline{\theta}_{1}=\underline{\theta}_{2}=\underline{\theta}$, say, and $g(x ; a, \underline{\theta})=h(y ; a, \underline{\theta})$,

$$
\widehat{P}= \begin{cases}1-\sum_{i=0}^{n-1}(-1)^{i} \frac{(n-1) !(m-1) !}{(n-i-1) !(m+i-1) !}\left(\frac{S}{T}\right)^{i} ; & T>S, \\ 1-\sum_{i=0}^{m-2}(-1)^{i} \frac{(n-1) !(m-1) !}{(n+i) !(m-i-2) !}\left(\frac{T}{S}\right)^{i+1}, & T<S .\end{cases}
$$

\section{Remarks 1 .}

(i) In the literature, the researchers have dealt with the estimation of $R(t)$ and " $P$," separately. If we look at the proofs of Theorem 5 and Theorem 6, we observe that the UMVUE of the sampled pdf is used to obtain the UMVUES of $R(t)$ and "P." Thus, we have established interrelationship between the two estimation problems.

(ii) In the literature, the researchers have derived the UMVUES of " $P$ " for the case when $X$ and $Y$ follow the same distribution (may be with different parameters). We have obtained UMVUES of " $P$ " for all the three situations, when $X$ and $Y$ follow the same distribution having all the parameters same other than $\alpha$ 's, when $X$ and $Y$ have the same distribution with different parameters and when $X$ and $Y$ have different distributions.

(iii) It follows from Lemma 2 that $\operatorname{Var}(\widehat{\alpha})=\alpha^{2} /(n-1) \rightarrow$ 0 as $n \rightarrow \infty$. Thus, $\widehat{\alpha}$ is a consistent estimator of $\alpha$. Since $\widehat{f}(x ; a, \alpha, \lambda, \underline{\theta}), \widehat{R}(t)$, and $\widehat{P}$ are continuous functions of consistent estimators, they are also consistent estimators of $f(x ; a, \alpha, \lambda, \underline{\theta}), R(t)$, and " $P$," respectively.

\section{MLES of $R(t)$ and " $P$ " When All the Parameters Are Unknown}

Looking at the distributions belonging to the family (1), we notice that, except for exponentiated Pareto and generalized exponentiated Pareto distributions $[(V)$ and $(X)], a=0$; that is, " $a$ " is known. For these two distributions $\underline{\theta}$ contains " $a$."
Irrespective of the distribution, the MLE of a is $\widetilde{a}=X_{(1)}=$ $\min _{1 \leq i \leq n} X_{i}$. From (3), the log-likelihood function is

$$
\begin{aligned}
L(a, \alpha, \lambda, \underline{\theta} \mid \underline{x})= & n \log \alpha+n \log \lambda+\sum_{i=1}^{n} \log g^{\prime}\left(x_{i} ; a, \underline{\theta}\right) \\
& -\lambda \sum_{i=1}^{n} g\left(x_{i} ; a, \underline{\theta}\right)+(\alpha-1) \\
& \times \sum_{i=1}^{n} \log \left(1-e^{-\lambda g\left(x_{i} ; a, \underline{\theta}\right)}\right) .
\end{aligned}
$$

First of all, we replace " $a$ " by $X_{(1)}$ in (22); then we differentiate (22) with respect to all the unknown parameters and equate these differential equations to zero. The MLES of unknown parameters are obtained on solving these equations simultaneously. Let $\widetilde{\alpha}, \widetilde{\lambda}$, and $\underline{\widetilde{\theta}}$ be the maximum likelihood estimators of $\alpha, \lambda$, and $\underline{\theta}$, respectively.

The following lemma provides The MLE of $f(x ; a, \alpha, \lambda, \underline{\theta})$ at a specified point " $x$ ".

Lemma 8. The MLE of $f(x ; a, \alpha, \lambda, \underline{\theta})$ at a specified point " $x$ " is

$\tilde{f}(x ; a, \alpha, \lambda, \underline{\theta})=\tilde{\alpha} \widetilde{\lambda} g^{\prime}(x ; \tilde{a}, \underline{\tilde{\theta}}) e^{-\tilde{\lambda} g(x ; \tilde{a}, \underline{\tilde{\theta}})}\left(1-e^{-\tilde{\lambda} g(x ; \tilde{a}, \tilde{\theta})}\right)^{\tilde{\alpha}-1}$.

Proof. The proof follows from (1) and the one-to-one property of the MLE.

In the following theorem, we derive the MLE of $R(t)$.

Theorem 9. The MLE of $R(t)$ is given by

$$
\widetilde{R}(t)=1-\left(1-e^{-\tilde{\lambda} g(t ; \tilde{a}, \underline{\theta})}\right)^{\tilde{\alpha}} .
$$

Proof. Using Lemma 8 and invariance property of the MLES,

$$
\begin{aligned}
\widetilde{R}(t) & =\int_{t}^{\infty} \tilde{f}(x ; a, \alpha, \lambda, \underline{\theta}) d x \\
& =\widetilde{\alpha} \widetilde{\lambda} \int_{t}^{\infty} g^{\prime}(x ; \widetilde{a}, \underline{\theta}) e^{-\tilde{\lambda} g(x ; \tilde{a}, \tilde{\theta})}\left(1-e^{-\tilde{\lambda} g(x ; \tilde{a}, \tilde{\theta})}\right)^{\tilde{\alpha}-1} d x \\
& =\widetilde{\alpha} \int_{\left(1-e^{-\tilde{\lambda} g(t ; \tilde{a}, \tilde{\theta})}\right)}^{1}(1-u)^{\widetilde{\alpha}-1} d u,
\end{aligned}
$$

and the theorem follows.

In the following theorem, we obtain the maximum likelihood estimator of " $P$." 
Theorem 10. The MLE of " $P$ " is given by

$$
\begin{aligned}
\widetilde{P}=\widetilde{\alpha}_{1} \int_{0}^{1} & {\left[1-\exp \left\{-\lambda_{2} \mathrm{~h}\left(\mathrm{~g}^{-1}\left\{-\boldsymbol{\lambda}_{1}^{-1} \log (1-\mathrm{u})\right\}\right)\right\}\right]^{\tilde{\alpha}_{2}} } \\
\times & u^{\widetilde{\alpha}_{1}-1} d u .
\end{aligned}
$$

Proof. Using Lemma 8 and the one-to-one property of the MLES

$$
\begin{aligned}
& \widetilde{P}=\int_{y=y_{(1)}}^{\infty} \int_{x=y}^{\infty} \tilde{f}_{1}\left(x ; a_{1}, \alpha_{1}, \lambda_{1}, \underline{\theta}_{1}\right) \\
& \times \tilde{f}_{2}\left(y ; a_{2}, \alpha_{2}, \lambda_{2}, \underline{\theta}_{2}\right) d x d y \\
&=\int_{y=y_{(1)}}^{\infty}\left[1-\widetilde{R}_{2}(x)\right] \tilde{f}_{1}\left(x ; a_{1}, \alpha_{1}, \lambda_{1}, \underline{\theta}_{1}\right) d x \\
&=\int_{y=y_{(1)}}^{\infty}\left[1-\exp \left\{-\tilde{\lambda}_{2} h\left(x ; \tilde{a}_{2}, \underline{\tilde{\theta}_{2}}\right)\right\}\right]^{\tilde{\alpha}_{2}} \\
& \quad \times \widetilde{\alpha}_{1} \tilde{\lambda}_{1} g^{\prime}\left(x ; \tilde{a}_{1}, \underline{\tilde{\theta}_{1}}\right) e^{-\tilde{\lambda}_{1} g^{\prime}\left(x ; \widetilde{a}_{1}, \underline{\tilde{\theta}_{1}}\right)} \\
& \times\left(1-e^{-\tilde{\lambda}_{1} g^{\prime}\left(x ; \tilde{a}_{1}, \underline{\tilde{\theta}_{1}}\right)}\right)^{\tilde{\alpha}-1} d x,
\end{aligned}
$$

and the result follows.

Corollary 11. For the case when $a_{1}=a_{2}=a$, say, $\lambda_{1}=\lambda_{2}=\lambda$, say, $\underline{\theta}_{1}=\underline{\theta}_{2}=\underline{\theta}$, say, and $g(x ; a, \underline{\theta})=h(y ; a, \underline{\theta})$,

$$
\widetilde{P}=\frac{\widetilde{\alpha}_{1}}{\widetilde{\alpha}_{1}+\widetilde{\alpha}_{2}}
$$

Remarks 2.

(i) All the comments made under Remarks 1 for UMVUES are tenable for MLES.

(ii) In the present approaches of obtaining UMVUES and MLES, one does not need the expressions of $R(t)$ and " $P$."

(iii) Since the UMVUES and MLES of powers of $\alpha$ are obtained under the same conditions, we compare their performances. For $q=-1$ the UMVUE and MLE of $\alpha$ are, respectively, $\widehat{\alpha}=(n-1)(-T)^{-1}$ and $\widetilde{\alpha}=(n)(-T)^{-1}$. For these estimators

$V(\widehat{\alpha})=\frac{\alpha^{2}}{(n-2)}, \quad V(\widetilde{\alpha})=\frac{n^{2}}{(n-1)^{2}(n-2)}$

Hence,

$$
V(\widetilde{\alpha})-V(\widehat{\alpha})=\frac{(2 n-1)}{(n-1)(n-2)} \alpha^{2}>0 .
$$

Thus, the UMVUE of $\alpha$ is more efficient than its MLE. Similarly, we can compare the performances of these estimators for other powers of $\alpha$.

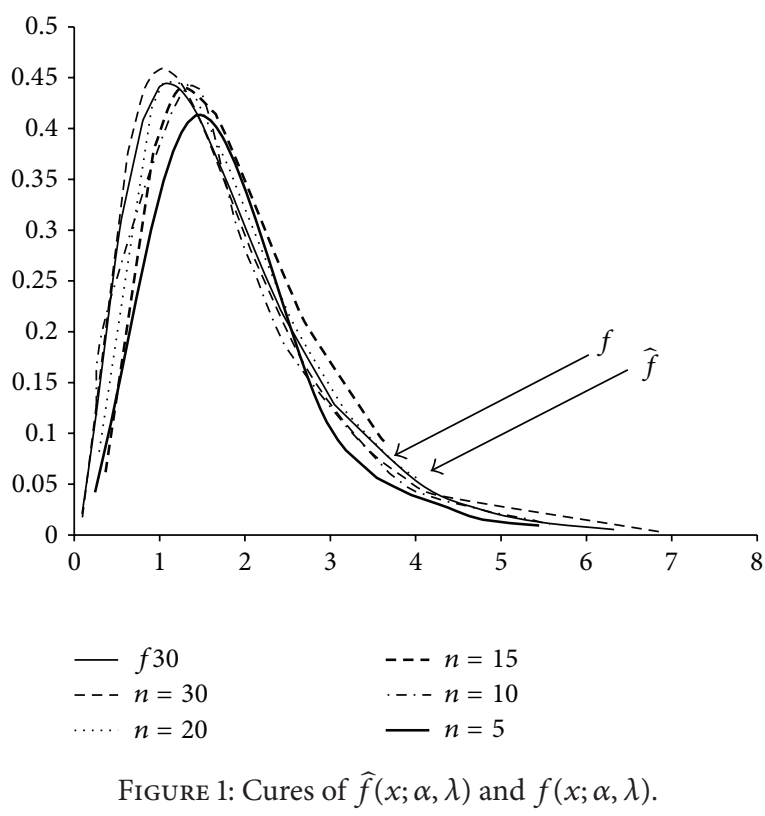

\section{Simulation Studies}

In order to verify the consistency of estimators, we have drawn samples of sizes $n=5,20$ and 30 from (1) with $g(x ; a, \underline{\theta})=x, a=0, \alpha=3$, and $\lambda=1$. In Figure 1 , we have plotted $\widehat{f}(x ; \alpha, \lambda)$ corresponding to these samples. We conclude that as the sample size increases, the curves of $\widehat{f}(x ; \alpha, \lambda)$ come closer to the curve of $f(x ; \alpha, \lambda)$, which is plotted in the same figure only for $n=30$. For $n=30$, the curves overlap. A similar pattern follows for the curves of $\widetilde{f}(x ; \alpha, \lambda)$.

For the case when $\alpha$ is unknown but other parameters are known, we have conducted simulation experiments using bootstrap resampling technique for sample sizes $n=5$, 10,20 , and 50 . The samples are generated from (1), with, $g(x ; a, \underline{\theta})=x, a=0, \alpha=3$, and $\lambda=1$. For different values of $t$, we have computed $\widehat{R}(t), \widetilde{R}(t)$, their corresponding bias, variance, 95\% confidence length, and corresponding coverage percentage. All the computations are based on 500 bootstrap replications and results are reported in Table 1.

In order to estimate " $P$," for the case when $\alpha_{1}$ and $\alpha_{2}$ are unknown but other parameters are known, we have conducted simulation experiments using bootstrap resampling technique for sample sizes $(n, m)=(5,5),(10,10),(15,15)$, $(25,25)$, and $(30,30)$. The samples are generated from (1), with $g\left(x ; a_{1}, \theta_{1}\right)=x, h\left(y ; a_{2}, \theta_{2}\right)=y, a_{1}=a_{2}=0, \lambda_{1}=$ $\lambda_{2}=1, \alpha_{1}=2$, and $\alpha_{2}=0.5(0.25) 1.25$. The computations are based on 500 bootstrap replications. We have computed $\widehat{P}, \widetilde{P}$, bias, variance, 95\% confidence length, and corresponding coverage percentage. The results are presented in Table 2.

In order to demonstrate the application of the theory developed in Section 4, we consider the following example. 
TABLE 1: Simulation results for $R(t)$.

\begin{tabular}{|c|c|c|c|c|c|c|c|c|c|}
\hline \multirow{2}{*}{$t$} & \multirow{2}{*}{$R(t)$} & \multicolumn{2}{|c|}{$n=5$} & \multicolumn{2}{|c|}{$n=10$} & \multicolumn{2}{|c|}{$n=20$} & \multicolumn{2}{|c|}{$n=50$} \\
\hline & & $\widehat{R}(t)$ & $\widetilde{R}(t)$ & $\widehat{R}(t)$ & $\widetilde{R}(t)$ & $\widehat{R}(t)$ & $\widetilde{R}(t)$ & $\widehat{R}(t)$ & $\widetilde{R}(t)$ \\
\hline \multirow{5}{*}{0.40} & \multirow{5}{*}{0.9642} & 0.9628 & 0.9429 & 0.9726 & 0.9638 & 0.9639 & 0.9598 & 0.9656 & 0.964 \\
\hline & & -0.0014 & -0.0213 & 0.0084 & $-4 e-04$ & $-2 e-04$ & -0.0044 & 0.0014 & $-1 e-04$ \\
\hline & & 0.001071326 & 0.001390097 & 0.0003646232 & 0.0003069262 & 0.0002067265 & 0.0002223408 & 0.0001467748 & 0.0001442984 \\
\hline & & 0.1211 & 0.1182 & 0.0647 & 0.0668 & 0.0555 & 0.0552 & 0.0477 & 0.0476 \\
\hline & & 86.6741 & 92.6193 & 91.428 & 93.112 & 93.5019 & 93.8349 & 94.0056 & 94.1243 \\
\hline \multirow{5}{*}{0.50} & \multirow{5}{*}{0.9391} & 0.8576 & 0.8546 & 0.8789 & 0.8769 & 0.9282 & 0.9252 & 0.9399 & 0.9386 \\
\hline & & -0.0815 & -0.0845 & -0.0601 & -0.0622 & -0.0109 & -0.0139 & $8 e-04$ & $-5 e-04$ \\
\hline & & 0.009229477 & 0.008767263 & 0.007167494 & 0.006830551 & 0.001421963 & 0.001408017 & 0.0005898798 & 0.0005745804 \\
\hline & & 0.1912 & 0.1524 & 0.2262 & 0.2086 & 0.1412 & 0.1371 & 0.0958 & 0.0947 \\
\hline & & 93.9509 & 94.0809 & 94.1651 & 94.4641 & 94.2804 & 94.6018 & 94.5567 & 94.6561 \\
\hline \multirow{5}{*}{0.80} & \multirow{5}{*}{0.8330} & 0.8314 & 0.8338 & 0.8355 & 0.8374 & 0.8408 & 0.8417 & 0.8319 & 0.8325 \\
\hline & & -0.0016 & $7 e-04$ & 0.0025 & 0.0044 & 0.0077 & 0.0087 & -0.0011 & $-5 e-04$ \\
\hline & & 0.004528085 & 0.002894221 & 0.002485577 & 0.002035995 & 0.002223323 & 0.002035827 & 0.001419582 & 0.001364689 \\
\hline & & 0.2552 & 0.2057 & 0.191 & 0.1725 & 0.1796 & 0.1711 & 0.1474 & 0.1446 \\
\hline & & 94.1964 & 94.3942 & 94.4812 & 94.5182 & 94.6424 & 94.6596 & 94.952 & 94.9534 \\
\hline \multirow{5}{*}{1.00} & \multirow{5}{*}{0.7474} & 0.729 & 0.7499 & 0.7936 & 0.7994 & 0.7279 & 0.7338 & 0.7548 & 0.7568 \\
\hline & & -0.0184 & 0.0025 & 0.0461 & 0.052 & -0.0195 & -0.0137 & 0.0074 & 0.0093 \\
\hline & & 0.008217478 & 0.005415503 & 0.005849085 & 0.005767851 & 0.004407818 & 0.003903546 & 0.002428885 & 0.002383391 \\
\hline & & 0.3395 & 0.2812 & 0.2359 & 0.2142 & 0.2505 & 0.2407 & 0.1915 & 0.1884 \\
\hline & & 94.0502 & 94.2394 & 94.5787 & 94.6276 & 94.9352 & 94.9729 & 95.0586 & 95.0578 \\
\hline \multirow{5}{*}{1.50} & \multirow{5}{*}{0.5311} & 0.4442 & 0.4933 & 0.5094 & 0.532 & 0.5283 & 0.5392 & 0.5256 & 0.53 \\
\hline & & -0.087 & -0.0378 & -0.0218 & $9 e-04$ & -0.0028 & 0.0081 & -0.0055 & -0.0011 \\
\hline & & 0.01260328 & 0.00618332 & 0.00520108 & 0.004460047 & 0.004379108 & 0.004317541 & 0.001922681 & 0.001875105 \\
\hline & & 0.2803 & 0.2719 & 80.2721 & 0.2648 & 0.26 & 0.2564 & 0.1747 & 0.1738 \\
\hline & & 94.2755 & 94.5729 & 94.464 & 94.653 & 94.7342 & 94.7791 & 95.0374 & 95.0517 \\
\hline \multirow{5}{*}{2.00} & \multirow{5}{*}{0.3535} & 0.4971 & 0.5442 & 0.3349 & 0.3573 & 0.3429 & 0.354 & 0.3485 & 0.3529 \\
\hline & & 0.1435 & 0.1906 & -0.0187 & 0.0037 & -0.0106 & $5 e-04$ & -0.005 & $-6 e-04$ \\
\hline & & 0.02667172 & 0.0416806 & 0.004913515 & 0.004757415 & 0.00239676 & 0.002330965 & 0.001298761 & 0.001284863 \\
\hline & & 0.3125 & 0.2928 & 0.2712 & 0.2765 & 0.1923 & 0.1943 & 0.1442 & 0.1448 \\
\hline & & 94.3591 & 94.7634 & 94.5513 & 94.7147 & 94.7592 & 94.8151 & 95.0453 & 95.0583 \\
\hline \multirow{5}{*}{2.50} & \multirow{5}{*}{0.2266} & 0.2396 & 0.2813 & 0.2699 & 0.2906 & 0.2047 & 0.2131 & 0.226 & 0.2295 \\
\hline & & 0.013 & 0.0547 & 0.0433 & 0.064 & -0.0219 & -0.0135 & $-6 e-04$ & 0.0029 \\
\hline & & 0.001733384 & 0.004863281 & 0.002956535 & 0.005265369 & 0.00116556 & 0.0009073532 & 0.0004148542 & 0.0004315035 \\
\hline & & 0.1576 & 0.1725 & 0.1312 & 0.1364 & 0.1045 & 0.1075 & 0.0809 & 0.0817 \\
\hline & & 94.0777 & 94.3206 & 94.2939 & 94.3724 & 94.7371 & 94.7634 & 95.0835 & 95.0883 \\
\hline
\end{tabular}

Here, the first row indicates the estimate, the second row indicates the bias, the third row indicates variance, the fourth row indicates $95 \%$ bootstrap confidence length, and the fifth row indicates the coverage percentage.

Let the $\mathrm{rv} X$ follow exponentiated exponential distribution with pdf

$$
f(x ; \alpha, \lambda, \delta)=\alpha \lambda \delta x^{\delta-1} e^{-\lambda x^{\delta}}\left(1-e^{-\lambda x^{\delta}}\right)^{\alpha-1} ; \quad x, \alpha, \lambda, \delta>0
$$

where $\alpha, \lambda$, and $\delta$ are unknown. Denoting, $L_{1}(\alpha, \lambda, \delta \mid x)$, the likelihood, the log-likelihood is given by

$$
\begin{aligned}
\log L_{1}(\alpha, \lambda, \delta x)= & n \log \alpha+n \log \lambda+n \log \delta \\
& +(\delta-1) \sum_{i=1}^{n} \log x_{i}-\lambda \sum_{i=1}^{n} x_{i}^{\delta} \\
& +(\alpha-1) \sum_{i=1}^{n} \log \left(1-e^{-\lambda x_{i}^{\delta}}\right) .
\end{aligned}
$$

Considering negative log-likelihood, then differentiating it with respect to all unknown parameters, and equating these differential coefficients to zero, from (32),

$$
\begin{gathered}
-\frac{n}{\alpha}-\sum_{i=1}^{n} \log \left(1-e^{-\lambda x_{i}^{\delta}}\right)=0, \\
-\frac{n}{\lambda}+\sum_{i=1}^{n} x_{i}^{\delta}-(\alpha-1) \sum_{i=1}^{n} \frac{x_{i}^{\delta} e^{-\lambda x_{i}^{\delta}}}{\left(1-e^{-\lambda x_{i}^{\delta}}\right)}=0, \\
-\frac{n}{\delta}-\sum_{i=1}^{n} \log x_{i}+\lambda \sum_{i=1}^{n} x_{i}^{\delta} \log x_{i}-(\delta-1) \lambda \\
\times \sum_{i=1}^{n} \frac{x_{i}^{\delta} e^{-\lambda x_{i}^{\delta}} \log x_{i}}{\left(1-e^{-\lambda x_{i}^{\delta}}\right)}=0 .
\end{gathered}
$$


TABLE 2: Simulation results for " $P$."

\begin{tabular}{|c|c|c|c|c|c|c|c|c|}
\hline \multirow{3}{*}{$\begin{array}{l}\left(\alpha_{1}, \alpha_{2}\right) \\
P \\
(n, m)\end{array}$} & \multicolumn{2}{|c|}{$(1,0.50)$} & \multicolumn{2}{|c|}{$(1,0.75)$} & \multicolumn{2}{|c|}{$(1,1.00)$} & \multicolumn{2}{|c|}{$(1,1.25)$} \\
\hline & \multicolumn{2}{|c|}{0.6667000} & \multicolumn{2}{|c|}{0.5714286} & \multicolumn{2}{|c|}{0.500000} & \multicolumn{2}{|c|}{0.4444000} \\
\hline & $\widetilde{P}$ & $\widehat{P}$ & $\widetilde{P}$ & $\widehat{P}$ & $\widetilde{P}$ & $\widehat{P}$ & $\widetilde{P}$ & $\widehat{P}$ \\
\hline \multirow{5}{*}{$(5,5)$} & 0.4160 & 0.4071 & 0.5196 & 0.5198 & 0.4937 & 0.4925 & 0.4503 & 0.4445 \\
\hline & -0.2507 & -0.2595 & -0.0518 & -0.0516 & -0.0063 & -0.0075 & 0.0058 & $1 e-04$ \\
\hline & 0.08305567 & 0.09161194 & 0.04060813 & 0.04711423 & 0.01738566 & 0.02128741 & 0.03447488 & 0.04041304 \\
\hline & 0.5225 & 0.5686 & 0.6963 & 0.7375 & 0.4996 & 0.5469 & 0.7179 & 0.7577 \\
\hline & 92.2467 & 91.7696 & 92.6155 & 91.9685 & 93.9423 & 93.6839 & 93.2283 & 92.5227 \\
\hline \multirow{5}{*}{$(10,10)$} & 0.4219 & 0.4179 & 0.5324 & 0.5340 & 0.5028 & 0.5029 & 0.4477 & 0.4451 \\
\hline & -0.2448 & -0.2487 & -0.0391 & -0.0374 & 0.0028 & 0.0029 & 0.0033 & $6 e-04$ \\
\hline & 0.06910139 & 0.07194417 & 0.01062704 & 0.01145625 & 0.01023848 & 0.01128198 & 0.01168588 & 0.01284482 \\
\hline & 0.3560 & 0.3727 & 0.3524 & 0.3698 & 0.3863 & 0.4050 & 0.4024 & 0.4213 \\
\hline & 93.4513 & 93.3550 & 93.5220 & 93.4724 & 94.1638 & 94.1400 & 93.2875 & 93.2019 \\
\hline \multirow{5}{*}{$(15,15)$} & 0.4507 & 0.4491 & 0.5601 & 0.5620 & 0.5015 & 0.5016 & 0.4499 & 0.4481 \\
\hline & -0.2159 & -0.2176 & -0.0113 & -0.0095 & 0.0015 & 0.0016 & 0.0054 & 0.0037 \\
\hline & 0.05200412 & 0.05309595 & 0.01157199 & 0.0122409 & 0.00717329 & 0.007664479 & 0.002471958 & 0.002628193 \\
\hline & 0.2739 & 0.2826 & 0.4016 & 0.4130 & 0.3234 & 0.3340 & 0.1911 & 0.1976 \\
\hline & 93.2657 & 93.2813 & 93.8776 & 93.8404 & 94.2206 & 94.1996 & 94.6752 & 94.6696 \\
\hline \multirow{5}{*}{$(25,25)$} & 0.4676 & 0.4670 & 0.5625 & 0.5637 & 0.4994 & 0.4994 & 0.4466 & 0.4455 \\
\hline & -0.1990 & -0.1997 & -0.0090 & -0.0077 & $-6 e-04$ & $-6 e-04$ & 0.0021 & 0.001 \\
\hline & 0.04415965 & 0.0446212 & 0.002974049 & 0.003065954 & 0.004487199 & 0.004669894 & 0.004399884 & 0.004568917 \\
\hline & 0.2529 & 0.2578 & 0.2048 & 0.2088 & 0.2579 & 0.2630 & 0.266 & 0.271 \\
\hline & 93.8839 & 93.87700 & 94.2551 & 94.2501 & 94.3152 & 94.3066 & 95.5079 & 95.4975 \\
\hline \multirow{5}{*}{$(30,30)$} & 0.4869 & 0.4867 & 0.5740 & 0.5753 & 0.4999 & 0.4999 & 0.447 & 0.4461 \\
\hline & -0.1798 & -0.1800 & 0.0026 & 0.0038 & $-1 e-04$ & $-1 e-04$ & 0.0025 & 0.0017 \\
\hline & 0.03621819 & 0.03642065 & 0.003972225 & 0.004105375 & 0.003633788 & 0.003756843 & 0.00493606 & 0.005091428 \\
\hline & 0.2470 & 0.2511 & 0.243 & 0.2468 & 0.2391 & 0.243 & 0.2797 & 0.2841 \\
\hline & 95.2267 & 95.2211 & 94.5145 & 94.5023 & 95.1531 & 95.1467 & 95.3624 & 95.3519 \\
\hline
\end{tabular}

Here, the first row indicates the estimate, the second row indicates the bias, the third row indicates variance, the fourth row indicates $95 \%$ bootstrap confidence length, and the fifth row indicates the coverage percentage.

The following sample of size 50 is generated from (31), for $\alpha=3.5, \lambda=1$, and $\delta=1$ as follows.

Sample 1. 1.4904, 0.5808, 2.2326, 0.9756, 2.3062, 1.4692, $1.0433,2.9527,0.9241,2.5901,1.2021,1.3022,3.6532,0.8146$, $1.7083,0.3126,2.4925,0.9462,0.4557,2.0120,1.6554,1.1651$, $0.9703,1.0305,0.4618,0.7465,1.9249,1.5763,1.9127,0.9742$, 2.7401, 1.3686, 1.8546, 2.3296, 1.4526, 1.1719, 4.4671, 1.1218, $1.1901,0.8248,3.0091,0.9511,1.3308,2.2145,2.0216,1.6740$, $1.5620,2.0339,0.6882,3.1335$.

Assuming that the data represents life spans of items in hours, $R(0.35)=0.986$, and solving (33) simultaneously, we get $\widetilde{\alpha}=3.711821, \widetilde{\lambda}=1.170381, \widetilde{\delta}=1.098677$, and $\widetilde{R}(0.35)=$ 0.9872 .

In order to obtain the maximum likelihood estimator of " $P$ ", we have generated one more sample of size 50 from (31), for $\alpha=3, \lambda=1$, and $\delta=1$ as follows.

Sample 2. 1.2271, 0.9467, 1.9800, 0.6613, 0.4526, 1.5701, $1.0414,1.9008,1.2378,3.7137,1.4012,0.5640,1.6704,2.0615$, $1.4360,1.6696,0.7443,0.9568,1.6306,0.2327,1.0024$, $0.8178, \quad 0.7704, \quad 2.1587, \quad 0.8460,2.3833, \quad 1.0702, \quad 1.1584$ $3.1261,3.7417, \quad 3.0401,2.4465,0.6874,3.2289, \quad 2.4881$,
$1.6636,0.4313,2.4856,1.2428,1.5750,1.1564,1.4370$, $3.1483,1.1472,1.4889,0.8209,0.6693,1.2684,3.2650$, 3.2285 .

For this sample, we have $\widetilde{\alpha}=2.932394, \tilde{\lambda}=1.044041$, and $\tilde{\delta}=1.106363$. Using these results (obtained from Samples 1 and 2 ) and Theorem 10 , we get $P=0.5384615$ and $\widetilde{P}=0.5586546$.

\section{Conclusions}

We propose a class of distributions, which covers as many as ten exponentiated distributions as special cases. The problems of estimating $R(t)$ and " $P$ " are considered. UMVUES and MLES are derived. A comparative study of the two methods of estimation is done. The estimators of " $P$ " are derived, which cover the cases when $X$ and $Y$ may follow the same, as well as, different distributions. By estimating the sampled pdf to obtain the estimators of $R(t)$ and " $P$," an interrelationship between the two estimation problems is established. Simulation study is performed, and a real-data example is presented. 


\section{Conflict of Interests}

The authors declare that there is no conflict of interests regarding the publication of this paper.

\section{Acknowledgment}

The authors are thankful to the referee for his valuable comments.

\section{References}

[1] G. S. Mudholkar and D. K. Srivastava, "Exponentiated Weibull family for analyzing bathtub failure-rate data," IEEE Transactions on Reliability, vol. 42, no. 2, pp. 299-302, 1993.

[2] D. I. Davis, "An analysis of some failure data," Journal of the American Statistical Association, vol. 47, pp. 113-150, 1952.

[3] B. Efron, "Logistic regression, survival analysis and the KaplanMeier curve," Journal of the American Statistical Association, vol. 83, pp. 414-425, 1988.

[4] G. S. Mudholkar and D. K. Srivasta, "Exponentiated weibull family: a reanalysis of the bus-motor-failure data," Technometrics, vol. 37, no. 4, pp. 436-445, 1995.

[5] G. S. Mudholkar and A. D. Hutson, "The exponentiated weibull family: some properties and a flood data application," Communications in Statistics-Theory and Methods, vol. 25, no. 12, pp. 3059-3083, 1996.

[6] R. Jiang and D. N. P. Murthy, "The exponentiated weibull family: a graphical approach," IEEE Transactions on Reliability, vol. 48, no. 1, pp. 68-72, 1999.

[7] M. M. Nassar and F. H. Eissa, "On the exponentiated weibull distribution," Communications in Statistics-Theory and Methods, vol. 32, no. 7, pp. 1317-1336, 2003.

[8] M. M. Nassar and F. H. Eissa, "Bayesian estimation for the exponentiated Weibull model," Communications in StatisticsTheory and Methods, vol. 33, no. 10, pp. 2343-2362, 2004.

[9] M. Pal, M. M. Ali, and J. Woo, "Exponentiated Weibull distribution," Statistica, vol. 66, no. 2, pp. 139-147, 2006.

[10] R. C. Gupta, P. L. Gupta, and R. D. Gupta, "Modeling failure time data by Lehman alternatives," Communications in Statistics-Theory and Methods, vol. 27, no. 4, pp. 887-904, 1998.

[11] R. D. Gupta and D. Kundu, "Generalized exponential distributions," Australian and New Zealand Journal of Statistics, vol. 41, no. 2, pp. 173-188, 1999.

[12] R. D. Gupta and D. Kundu, "Generalized exponential distribution: different method of estimations," Journal of Statistical Computation and Simulation, vol. 69, no. 4, pp. 315-337, 2001.

[13] R. D. Gupta and D. Kundu, "Exponentiated exponential family: an alternative to gamma and Weibull distributions," Biometrical Journal, vol. 43, pp. 117-130, 2001.

[14] R. D. Gupta and D. Kundu, "Generalized exponential distributions: statistical inference," Journal of Statistical Theory and Applications, vol. 1, pp. 101-118, 2002.

[15] R. D. Gupta and D. Kundu, "Discriminating between Weibull and generalized exponential distributions," Computational Statistics and Data Analysis, vol. 43, no. 2, pp. 179-196, 2003.

[16] R. D. Gupta and D. Kundu, "Closeness of gamma and generalized exponential distribution," Communications in StatisticsTheory and Methods, vol. 32, no. 4, pp. 705-721, 2003.
[17] R. D. Gupta and D. Kundu, "Discriminating between gamma and generalized exponential distributions," Journal of Statistical Computation and Simulation, vol. 74, no. 2, pp. 107-121, 2004.

[18] R. D. Gupta, D. Kundu, and A. Manglick, "Probability of correct selection of gamma versus GE or Weibull versus GE based likelihood ratio statistic," in Proceedings of Statistics Recent Advances in Statistical Methods, Y. P. Chaubey, Ed., pp. 147-156, Imperial College Press, 2002.

[19] D. Kundu, R. D. Gupta, and A. Manglick, "Discriminating between the log-normal and generalized exponential distributions," Journal of Statistical Planning and Inference, vol. 127, no. 1-2, pp. 213-227, 2005.

[20] D. Kundu and R. D. Gupta, "Estimation of $\mathrm{P}[Y<X]$ for generalized exponential distribution," Metrika, vol. 61, no. 3, pp. 291-308, 2005.

[21] M. Z. Raqab and M. Ahsanullah, "Estimation of the location and scale parameters of generalized exponential distribution based on order statistics," Journal of Statistical Computation and Simulation, vol. 69, no. 2, pp. 109-123, 2001.

[22] M. Z. Raqab, "Inferences for generalized exponential distribution based on record statistics," Journal of Statistical Planning and Inference, vol. 104, no. 2, pp. 339-350, 2002.

[23] A. H. Abdel-Hamid and E. K. AL-Hussaini, "Estimation in stepstress accelerated life tests for the exponentiated exponential distribution with type-I censoring," Computational Statistics and Data Analysis, vol. 53, no. 4, pp. 1328-1338, 2009.

[24] E. K. AL-Hussaini, "On exponentiated class of distributions," Journal of Statistical Theory and Applications, vol. 8, pp. 41-63, 2010.

[25] E. K. AL-Hussaini and M. Hussein, "Estimation using censored data from exponentiated Burr type XII population," American Open Journal of Statistics, vol. 1, pp. 33-45, 2011.

[26] J. F. Lawless, Statistical Models and Methods for Lifetime Data, Wiley, New York, NY, USA, 2nd edition, 2003.

[27] A. I. Shawky and H. H. Abu-Zinadah, "Exponentiated Pareto distribution: different method of estimations," International Journal of Contemporary Mathematical Sciences, vol. 4, no. 14, pp. 677-693, 2009.

[28] G. M. Cordeiro, G. O. Silva, and E. M. M. Ortega, "The beta extended weibull family," Journal of Probability and Statistical Science, vol. 10, pp. 15-40, 2012.

[29] D. Kundu and M. Z. Raqab, "Generalized Rayleigh distribution: different methods of estimations," Computational Statistics and Data Analysis, vol. 49, no. 1, pp. 187-200, 2005.

[30] I. B. Abdul-Moniem and H. F. Abdel-Hameed, "On exponentiated Lomax distribution," International Journal of Mathematical Archive, vol. 3, no. 5, pp. 2144-2150, 2012.

[31] P. R. Tadikamalla, "A look at the Burr and related distributions," International Statistical Review, vol. 48, pp. 337-344, 1980.

[32] C. D. Lai, M. Xie, and D. N. P. Murthy, "A modified Weibull distribution," IEEE Transactions on Reliability, vol. 52, no. 1, pp. 33-37, 2003.

[33] M. Xie, Y. Tang, and T. N. Goh, "A modified Weibull extension with bathtub-shaped failure rate function," Reliability Engineering and System Safety, vol. 76, no. 3, pp. 279-285, 2002.

[34] M. Ljubo, "Curves and concentration indices for certain generalized Pareto distributions," Statistical Review, vol. 15, pp. 257260, 1965.

[35] V. K. Rohatgi, An Introduction to Probability Theory and Mathematical Statistics, John Wiley \& Sons, New York, NY, USA, 1976. 
[36] N. L. Johnson and S. Kotz, Continuous Univariate DistributionsI, John Wiley \& Sons, New York, NY, USA, 1970.

[37] A. Chaturvedi and S. K. Tomer, "Classical and Bayesian reliability estimation of the negative binomial distribution," Journal of Applied Statistical Science, vol. 11, pp. 33-43, 2002. 


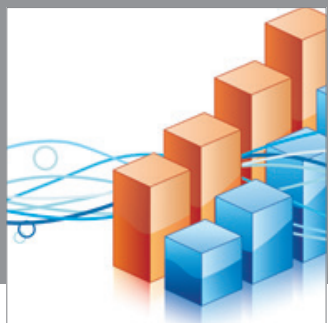

Advances in

Operations Research

mansans

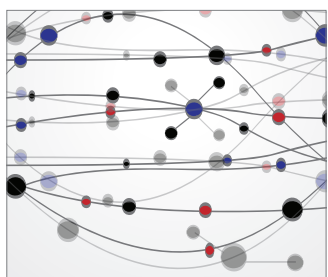

The Scientific World Journal
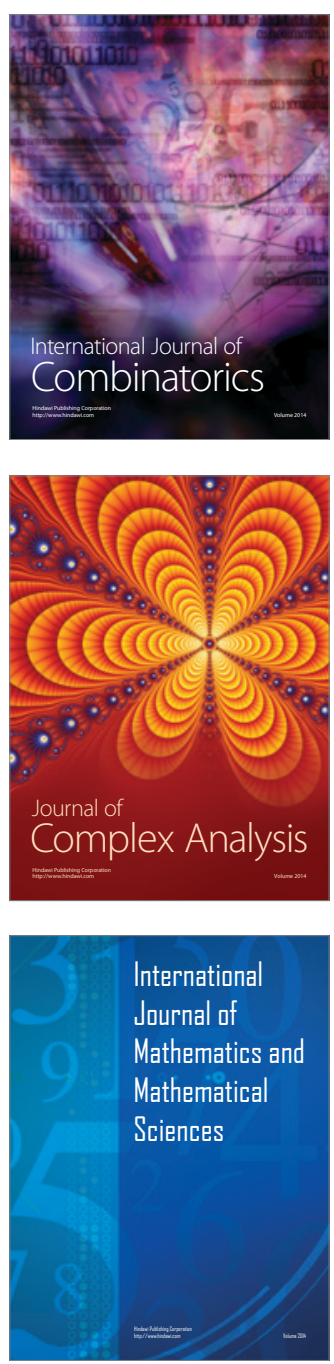
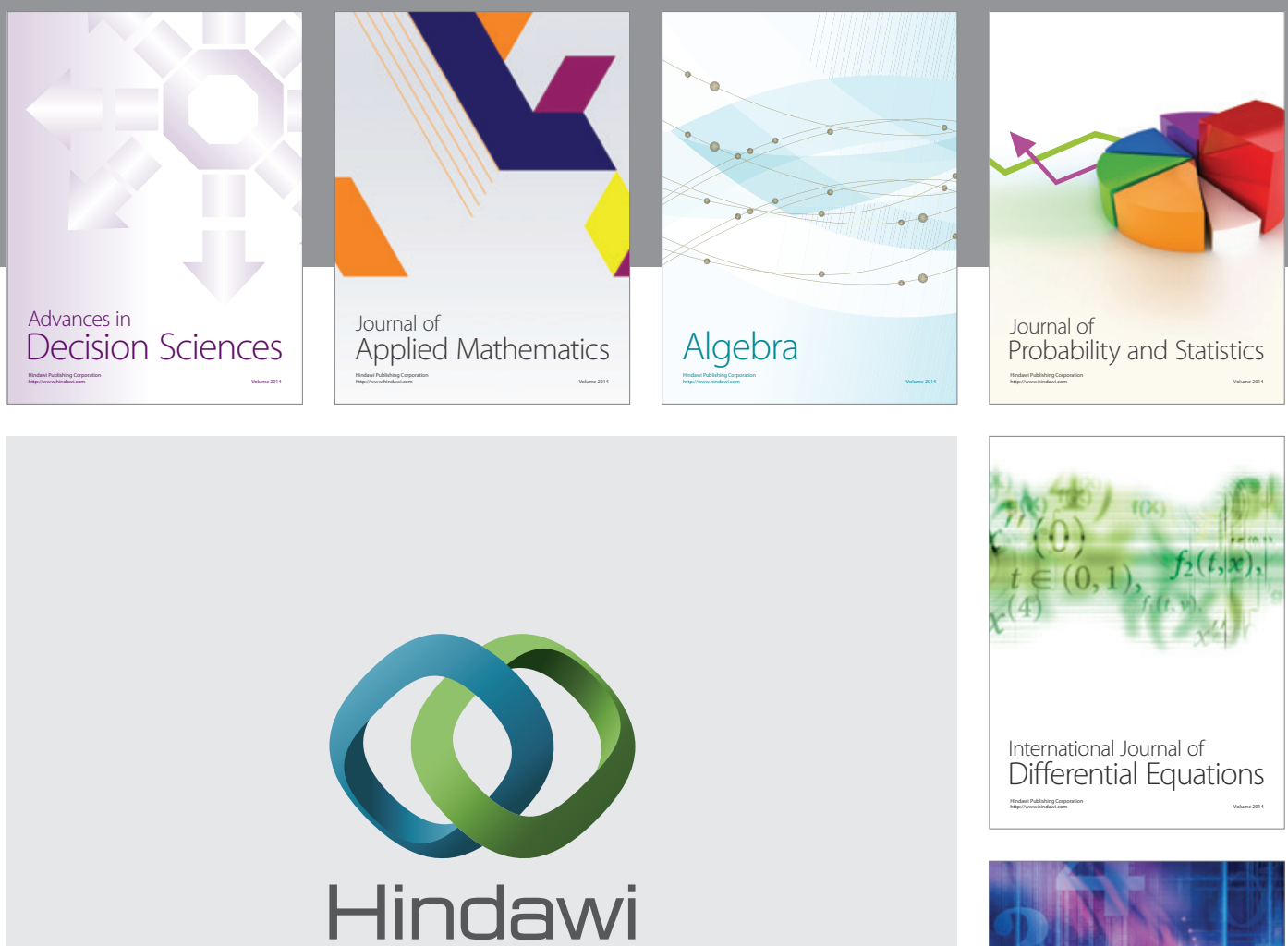

Submit your manuscripts at http://www.hindawi.com
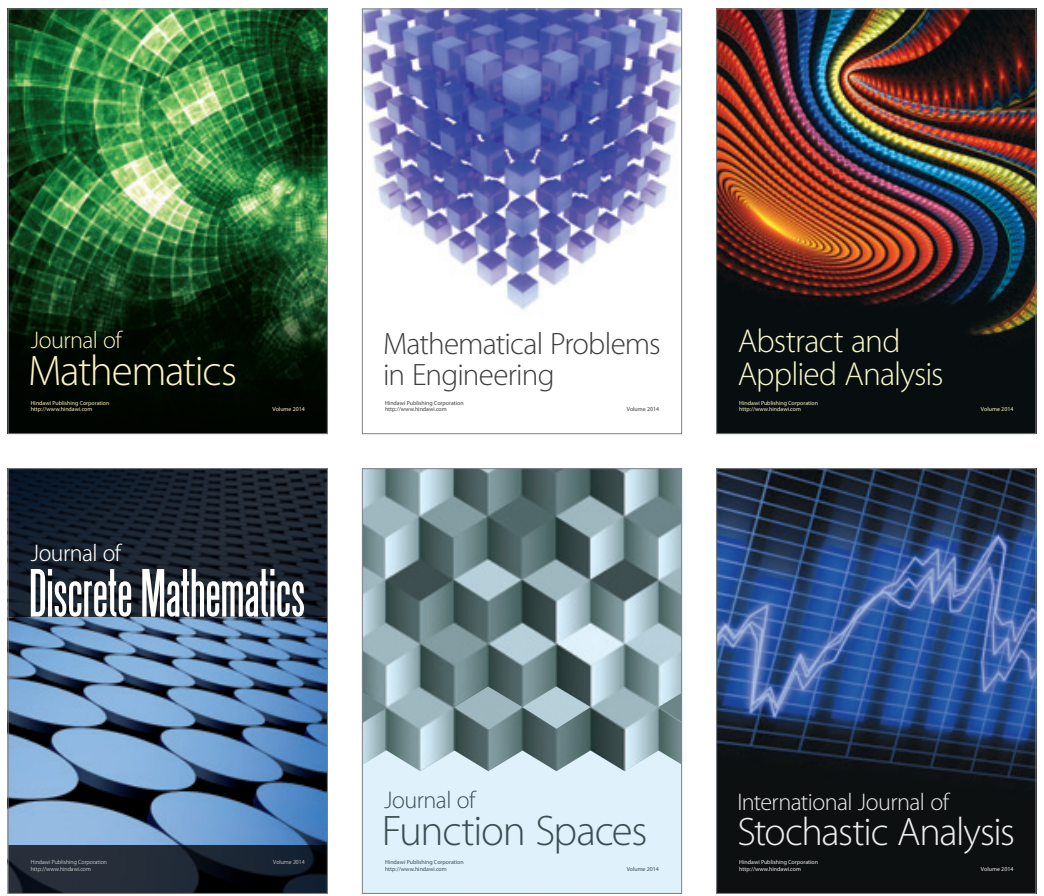

Journal of

Function Spaces

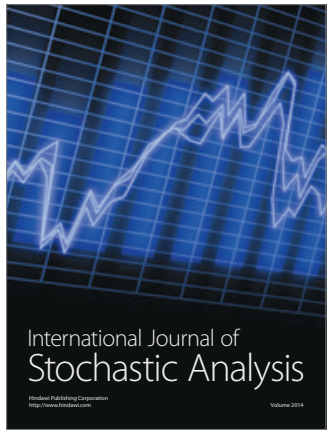

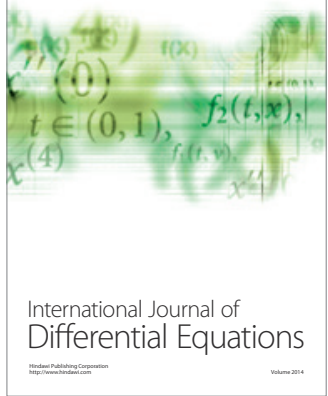
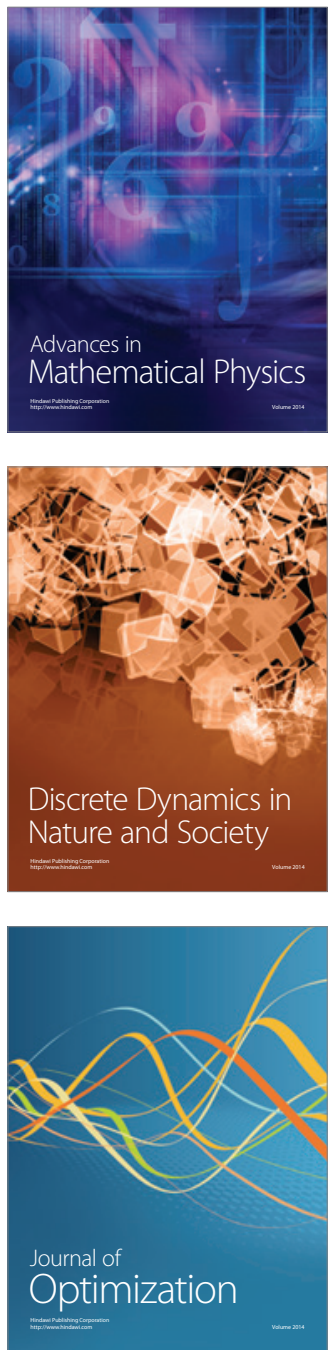